\title{
Métallurgie des phases icosaédriques
}

Y. Calvayrac

CECM-CNRS, 15 rue Georges Urbain, 94707 Vitry-sur-Seine cedex, France

\begin{abstract}
Quasicrystalline phases have been discovered in numerous alloy systems. This paper gives a short introductory review on some aspects of quasicrystal metallurgy. From metastable quasicrystal phases to highly perfect icosahedral single grains, spectacular progress in the structural quality of the samples has led to understand how quasicrystal behaviour differs from that of other phases. This knowledge now is used to optimize the specific characteristics in the field of the electronic properties.
\end{abstract}

\section{INTRODUCTION}

Plusieurs ouvrages très complets traitent de l'état actuel des connaissances dans le domaine des quasicristaux [1] [2] [3]. Le présent exposé se bornera à un survol de l'évolution du sujet sous son aspect métallurgique et montrera comment l'amélioration de la qualité métallurgique des échantillons a permis et conditionne encore le développement du thème.

Très rapidement après la découverte [4] des étapes cruciales ont été franchies : dès 1985 l'application de la méthode de coupe et projection [5] montrait la voie à suivre pour simplifier les concepts et progresser dans l'étude de la structure, en raisonnant dans un espace de dimension supérieure où toute l'information expérimentale se retrouve dans la maille d'un réseau périodique. Presque simultanément le problème - si important - de l'indexation des diagrammes de diffraction était résolu [6]. En 1988 la première fonction de Patterson d'un quasicristal [7] montrait combien la structure icosaédrique de (Al,Si)-Mn était simple... dans l'espace de dimension 6.

Au niveau du matériau, l'étape décisive a certainement été la découverte, dans le système $\mathrm{Al}-\mathrm{Cu}-\mathrm{Fe}$ [8], de la première phase icosaédrique thermodynamiquement stable que l'on puisse qualifier de "parfaite" en ce sens que la largeur des pics de diffraction haute résolution est égale à la largeur instrumentale. L'existence de cette phase a démontré que le désordre dans les quasicristaux n'était pas universel, et a révélé des propriétés spécifiques masquées jusqu'alors par les défauts structuraux.

\section{LA PERFECTION DES PHASES ICOSAÉDRIQUES}

Les premiers quasicristaux découverts sont métastables, ils ont une forte densité de défauts, et les échantillons ne sont pas monophasés. Ils appartiennent essentiellement aux systèmes binaires Al-T où $\mathrm{T}$ représente Mn (l'alliage de la découverte), Cr, Ru, V, W ou Mo, et ils sont préparés par hypertrempe depuis l'état liquide, comme les amorphes métalliques.

Dans les alliages Al-Mn rapidement solidifiés, la phase icosaédrique fournit les sites de germination d'une phase décagonale qui prédomine dès que la vitesse de refroidissement diminue. L'addition de Si a permis d'éliminer la phase décagonale et des échantillons icosaédriques pratiquement monophasés, plus stables (température de transformation plus élevée) et de meilleure qualité structurale ont été obtenus. La composition la plus étudiée est $\mathrm{Al}_{73} \mathrm{Si}_{6} \mathrm{Mn}_{21}$ qui semble cependant impossible à obtenir sans traces d'Al et de la phase $\beta$-Al-Mn-Si (les phases d'équilibre à $700^{\circ} \mathrm{C}$ ). Des études récentes [9] montrent que la composition la plus favorable serait $\mathrm{Al}_{74} \mathrm{Si}_{4} \mathrm{Mn}_{22}$, seule compositon où une phase $\mathrm{i}-\mathrm{Al}-\mathrm{Mn}$-Si strictement monophasée ait été observée. 
A une composition proche, la phase périodique stable $\alpha$-Al-Mn-Si a des caractéristiques structurales qui la rapprochent de la phase icosaédrique: cette phase (cubique presque centrée, $a=12,7 \AA$ ) contient des amas de 54 atomes à coordination icosaédrique (connus sous le nom d'icosaèdres de Mackay) qui ont été utilisés dès 1985 pour construire un modèle structural de la phase i-Al-Mn [10]. A côté de cet aspect structural, l'existence d'un approximant périodique de la phase icosaédrique était également importante pour comparer les caractéristiques physiques et mettre en évidence d'éventuelles propriétés liées à la quasipériodicité. La phase $\alpha$-Al-Mn-Si est difficile à obtenir sans traces d'aluminium. La composition la plus favorable est $\mathrm{Al}_{72,5} \mathrm{Si}_{10,1} \mathrm{Mn}_{17,4}$ mais la comparaison avec i-Al-Mn-Si reste difficile à cause de la mauvaise qualité structurale de la phase icosaédrique: l'état structural des phases icosaédriques métastables ne se perfectionne pas par recuit (même dans le cas de i-Al-V-Cu dont la température de transformation peut atteindre $95 \%$ du point de fusion!), et la présence des défauts modifie profondément les caractéristiques physiques.

Préparés par hypertrempe, les échantillons des phases icosaédriques métastables se présentent sous la forme de paillettes d'environ $30 \mu \mathrm{m}$ d'épaisseur et de quelques $\mathrm{mm}^{2}$ de surface. L'identification d'une phase icosaédrique thermodynamiquement stable dans le système $\mathrm{Al}-\mathrm{Li}$-Cu [11] a permis de disposer enfin d'échantillons massifs et de faire croître des monograins de taille de l'ordre du $\mathrm{cm}^{3}$. Le spectre de diffraction de $\mathrm{i}-\mathrm{Al}_{6} \mathrm{Li}_{3} \mathrm{Cu}$, et la structure de la phase approximante $\mathrm{R}-\mathrm{Al}{ }_{5} \mathrm{Li} 3 \mathrm{Cu}$ bâtie avec des amas icosaédriques "de Bergman", différents de ceux de Mackay, ont montré qu'il existait au moins deux classes de quasicristaux, de structure atomique différente. La structure de la phase $\mathrm{R}$ (type $\left.\mathrm{Mg}_{32}(\mathrm{Zn}, \mathrm{Al})_{49}\right)$ a inspiré le modèle structural proposé par Audier et al. [12] pour la phase i-Al-Li-Cu.

Mais d'importantes difficultés métallurgiques empêchent d'obtenir i- $\mathrm{Al}_{6} \mathrm{Li}_{3} \mathrm{Cu}$ dans un état de grande perfection structurale: la formation par réaction péritectique entraîne des ségrégations macroscopiques très importantes dans l'alliage massif obtenu par les techniques classiques de solidification. Les traitements thermiques prolongés nécessaires à l'homogénéisation sont handicapés par le caractère très réactif et volatil du lithium. On peut cependant faire crôttre des monograins, par solidification lente à partir d'un alliage enrichi en aluminium. Les meilleurs résultats ont été obtenus en confinant le lithium lors de tous les traitements thermiques [13].

La mauvaise qualité structurale de cette phase stable laissait penser que le désordre était un caractère intrinsèque de ces matériaux, ce qui permettait de proposer d'autres modèles que l'ordre translationnel quasipériodique pour décrire la structure: par exemple, le modèle de "verre icosaédrique" [14], constitué d'un empilement aléatoire d'amas icosaédriques préservant un ordre orientationnel à longue distance, qui donne un spectre de diffusion avec des pics relativement étroits dont la largeur ne dépend que de la composante orthogonale du vecteur d'onde. L'obtention, dans les systèmes $\mathrm{Al}$-Cu-Fe et $\mathrm{Al}$-Cu-Ru, de phases icosaédriques thermodynamiquement stables parfaitement ordonnées a démontré que des cristaux à ordre translationnel quasipériodique existaient vraiment.

L'existence de ces phases pose immédiatement la question de l'origine de leur stabilité et, à côté du schéma quasipériodique "idéal" où la structure est parfaitement quasipériodique à l'état fondamental, des modèles de "pavage aléatoire" ont été proposés: Une énergie interne un peu plus basse favoriserait le cristal qui serait l'état fondamental. A température suffisamment élevée le quasicristal deviendrait l'état d'équilibre thermique grâce à la (faible) contribution supplémentaire de l'entropie de configuration liée au caractère aléatoire du pavage permis par les règles d'empilement [15]. L'analyse des profils des raies de diffraction en fonction de la température peut peut-être permettre de trancher entre les deux modèles. Quoi qu'il en soit, les positions des pics de Bragg dans l'espace réciproque sont strictement les mêmes et la plupart des propriétés seront les mêmes.

La phase i-Al-Cu-Fe se forme par réaction péritectique et il est donc difficile d'obtenir, par les techniques classiques, des échantillons massifs monophasés et homogènes. L'hypertrempe depuis l'état liquide est donc encore utilisée ici, bien que la phase soit stable, pour préparer des échantillons homogènes de composition strictement définie. Les échantillons bruts d'hypertrempe contiennent des défauts et une faible proportion d'une seconde phase (cubique ordonnée de type AlFe-B2, a = 2,9 $\AA$ ) qui s'éliminent facilement par recuit.

L'excellente qualité structurale de ce type d'échantillons a permis d'aborder l'étude des défauts par microscopie électronique en transmission: des dislocations ont été mises en évidence, révélant des propriétés de contraste spécifiques, sans équivalent dans les cristaux, induites par la composante perpendiculaire non nulle du vecteur de faute [16]. Cette première étude systématique de la topologie d'un défaut linéique dans un quasicrystal a été effectuée en extrayant les différentes composantes de Fourier d'images haute résolution digitalisées. Elle a montré que les dislocations quasicristallines doivent être définies par un vecteur de Burgers à 6 dimensions, ce qui signifie qu'un champ de déplacement doit leur être associé non seulement dans l'espace physique mais aussi dans l'espace complémentaire (appelé 
ici "espace perpendiculaire"). Ce champ de "phasons" correspond à une réorganisation locale des amas atomiques constituant le pavage quasipériodique.

Des défauts analogues aux parois d'antiphase des cristaux usuels ont aussi été observés, dans des échantillons $\mathrm{Al}-\mathrm{Cu}-\mathrm{Fe}$ bruts d'hypertrempe. Le diagramme de diffraction de $\mathrm{i}$-Al-Cu-Fe montre que sa structure correspond, dans un espace de dimension 6, à un réseau hypercubique à faces centrées, l'un des trois réseaux compatibles avec la symétrie icosaédrique. L'observation de parois d'antiphase [17] indique que cette structure peut se décrire comme une surstructure d'un réseau primitif hypercubique correspondant à une mise en ordre $\mathrm{P}(\mathrm{A}) \rightarrow \mathrm{F}(2 \mathrm{~A})$ où $\mathrm{A}$ est le paramètre du réseau primitif $\mathrm{P}$ à 6 dimensions: il y a deux variantes possibles d'où l'existence de domaines ordonnés séparés par des parois (figure 1) dont la topologie est identique à celle des alliages ordonnés de type B2. Cet exemple montre la puissance de la représentation des défauts dans l'espace de dimension 6 , qui permet de faire l'analogie avec les cristaux périodiques tout en révélant la nature quasipériodique du matériau: l'analyse du contraste des parois d'antiphase montre en effet que la composante perpendiculaire du vecteur de faute n'est pas nulle, la paroi n'est pas une simple translation relative des deux domaines ordonnés, un réarrangement intervient dans l'empilement relatif des amas atomiques.

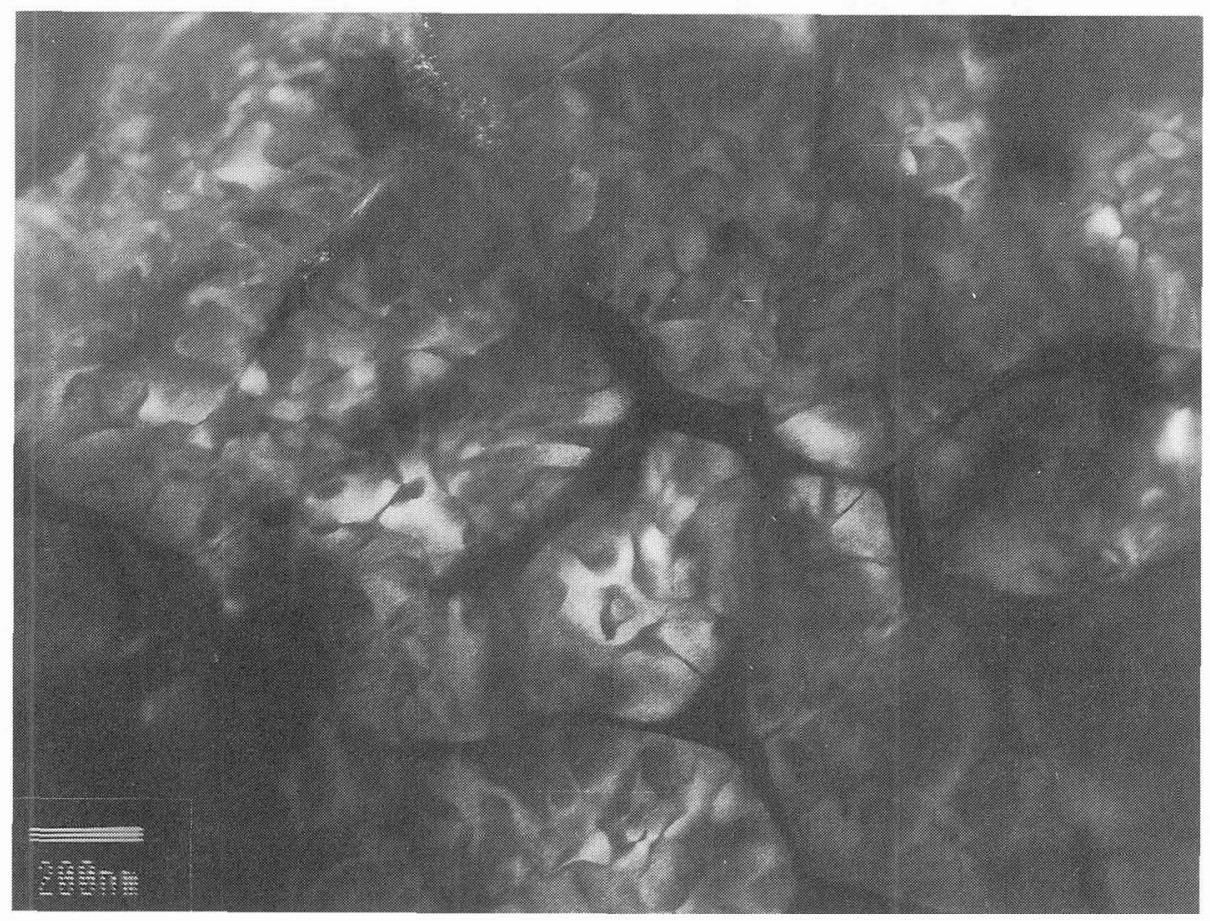

Figure 1: Parois d'antiphase dans la phase i-Al-Cu-Fe (alliage $\mathrm{Al}_{65} \mathrm{Cu}_{20} \mathrm{Fe}_{15}$ brut d'hypertrempe). Une seconde phase (type $\beta$-AlFe) est visible dans les espaces interdendritiques. (Avec la permission de J. Devaud, CECM / CNRS Vitry.)

Des monograins icosaédriques $\mathrm{Al}-\mathrm{Cu}-\mathrm{Fe}$ peuvent être obtenus, par croissance dans le liquide endessous du péritectique (figure 2). Ces monograins sont d'excellente qualité structurale mais leur taille limite est de l'ordre de quelques $\mathrm{mm}$. La localisation, dans le diagramme de phases Al-Pd-Mn, d'un domaine de compositions où une phase icosaédrique stable précipite directement dans le liquide, sans passer par la réaction péritectique [18], a permis de faire croître des monograins parfaits d'1 cm de diamètre et de plusieurs $\mathrm{cm}$ de long [19]: de tels échantillons (figure 3) allaient faire progresser de façon spectaculaire l'étude des propriétés mécaniques.

La phase i-Al-Pd-Mn stable et parfaite existe dans un petit domaine de compositions, autour de $\mathrm{Al}_{70} \mathrm{Pd}_{22} \mathrm{Mn} 8$. Dans ce domaine l'état structural est parfait même dans les échantillons bruts 
d'hypertrempe. La surface de cristallisation primaire a été récemment précisée par Gödecke et Lück [20] dans un article qui fournit les données les plus complètes et les plus fiables disponibles actuellement sur le diagramme de phases ternaire. Les monograins sont préparés par les techniques classiques de Bridgman ou de Czochralski, à partir d'un alliage liquide de composition située dans la zone de cristallisation primaire. Comme la solidification n'est pas congruente la méthode de Czochralski est préférable pour obtenir des échantillons homogènes de taille importante.

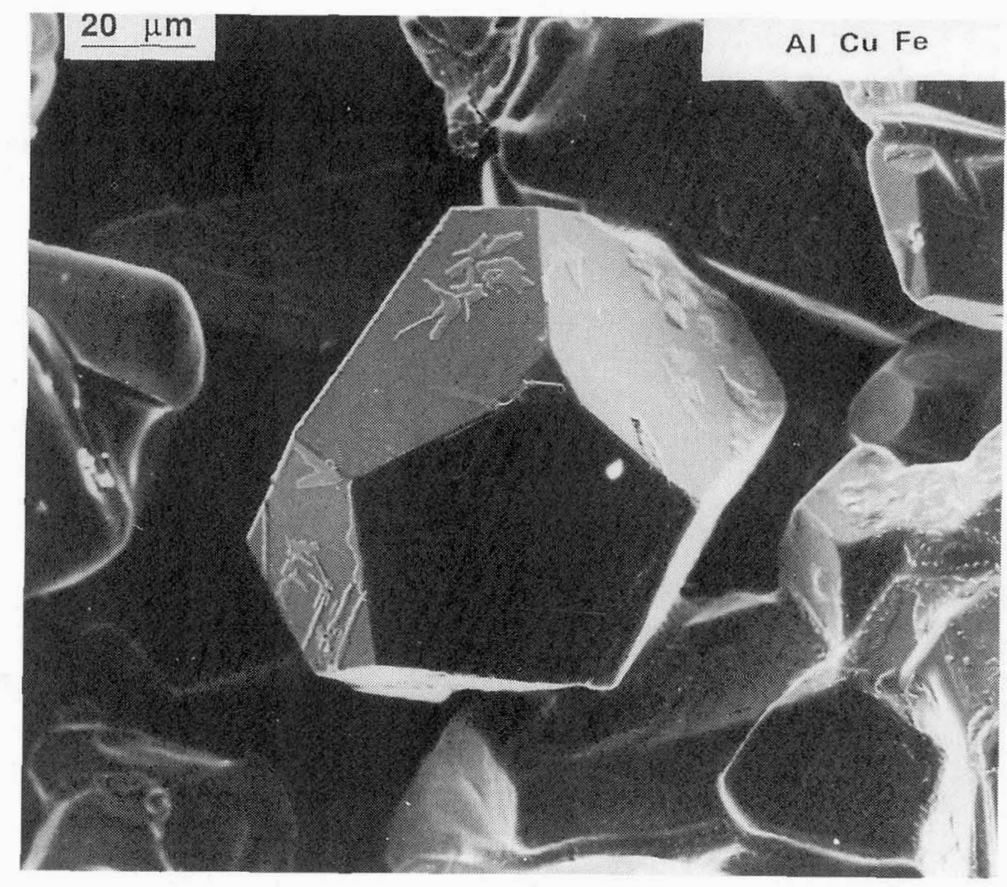

Figure 2: Monograin dodécaédrique i-Al-Cu-Fe obtenu par recuit prolongé à $850^{\circ} \mathrm{C}$. (Avec la permission de $\mathrm{A}$. Quivy, CECM / CNRS Vitry.)

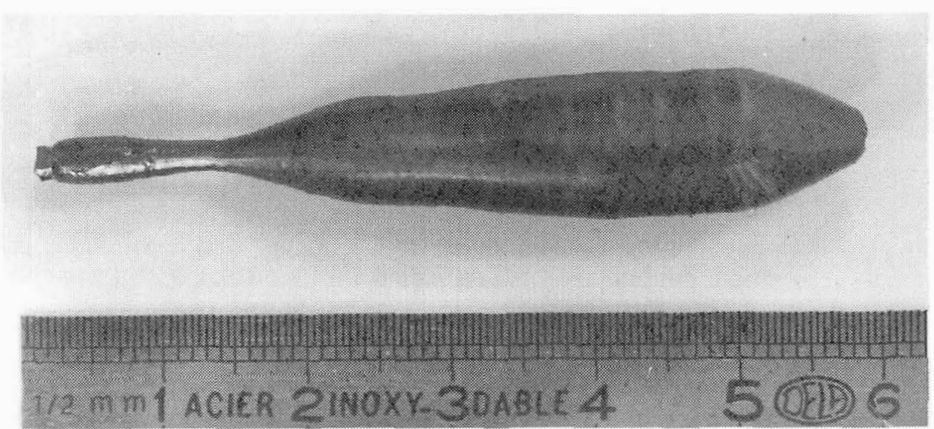

Figure 3: Monograin i-Al-Pd-Mn obtenu par la méthode de Czochralski. (Avec la permission de C. Prévost, CECM / CNRS Vitry.) 
La mosaïcité des monograins icosaédriques Al-Pd-Mn rivalise avec celle des meilleurs cristaux de silicium. Certains monograins ont une densité de défauts si faible que des effets de diffraction dynamique des rayons X tels que l'effet Borrmann peuvent être observés [21].

Les largeurs des pics de Bragg des diagrammes de diffraction des rayons $\mathrm{X}$ montrent que la longueur de cohérence, dans les phases icosaédriques Al-Cu-Fe, Al-Cu-Ru, Al-Pd-Mn et Al-Pd-Re, excède couramment le micron. Une telle perfection structurale a permis de mettre en évidence des caractéristiques électriques étonnantes qui atteignent, dans i-Al-Pd-Re, des valeurs exceptionnelles, et font de ces alliages une nouvelle classe de matériaux: bien que constitués de bons métaux, ils ont, au regard de la résistivité, un comportement de semi-conducteur dopé...

\section{CONSÉQUENCES SUR LES PROPRUÉTÉS PHYSIQUES}

\subsection{La résistivité des phases icosaédriques}

Dans les phases icosaédriques métastables les valeurs de la résistivité ( $\rho)$ sont de l'ordre de grandeur de celles des amorphes métalliques (100 à $400 \mu \Omega \mathrm{cm}$ ). Des valeurs plus élevées sont atteintes dans les quasicristaux stables imparfaits: dans les rubans hypertrempés de la phase i-Al-Li-Cu, $\rho$ se situe dans le domaine 500-1000 $\mu \Omega \mathrm{cm}$, selon la qualité structurale des échantillons, dans les monograins $\rho$ est de l'ordre de $800 \mu \Omega \mathrm{cm}$ à température ambiante. Dans les phases icosaédriques stables et parfaites la valeur de $\rho$ augmente, en même temps que le rapport résistif $\rho_{4,2 \mathrm{~K}} / \rho_{300 \mathrm{~K}}$, et devient extrêmement sensible à la composition: $\rho 4 \mathrm{~K}$ atteint $10000 \mu \Omega \mathrm{cm}$ dans $\mathrm{i}-\mathrm{Al}_{62,5} \mathrm{Cu}_{25} \mathrm{Fe}_{12,5}$ [22] et i- $\mathrm{Al}_{70,5} \mathrm{Pd}_{22} \mathrm{Mn}_{7,5}$ [23], et 30000 $\mu \Omega \mathrm{cm}$ dans i- $\mathrm{Al}_{65} \mathrm{Cu}_{20} \mathrm{Ru}_{15}$ où le rapport $\rho_{4 \mathrm{~K}} / \rho_{300 \mathrm{~K}}$ est voisin de 4 [24]. Pour comparaison, rappelons que les métaux constituant ces alliages ont des résistivités à température ambiante de quelques $\mu \Omega \mathrm{cm}$ qui diminuent quand la température diminue ou quand l'état structural s'améliore.

Dès 1991, l'analyse des résultats obtenus sur i-Al-Cu-Fe et i-Al-Cu-Ru à $4 \mathrm{~K}$ et à $300 \mathrm{~K}$ a conduit Klein et al. [22] à envisager la proximité d'une transition métal-isolant. Des résultats récents montrent la justesse de cette prévision: des valeurs de $\rho_{4 K}$ supérieures à $10 \Omega \mathrm{cm}$ et des rapports $\rho_{4 K} / \rho_{300 K}$ supérieurs à 20 ont été mesurés dans $i$-Al-Pd-Re [25], la résistivité électrique de ces quasicristaux est comparable à celle des semi-conducteurs dopés! Mais il faut préciser que ces valeurs chutent d'un facteur $10^{4}$ quand la composition ou le traitement thermique change [26]... La maîtrise des propriétés physiques sera donc un défi pour la métallurgie.

\subsection{La maîtrise des propriétés physiques: aspect métallurgique}

Un traitement thermique adéquat, une composition chimique bien choisie et bien contrôlée - c'est à dire une bonne connaissance du diagramme de phases - sont nécessaires pour comprendre et pour optimiser les propriétés physiques intrinsèques des quasicristaux.

\subsubsection{Effet du désordre structural et des phases parasites}

Les échantillons icosaédriques bruts d'hypertrempe contiennent des défauts (dislocations, fautes dans l'empilement quasipériodique des amas atomiques) qui abaissent la résistivité: i- $\mathrm{Al}_{70,5} \mathrm{Pd}_{22} \mathrm{Mn}_{7,5}$, dont l'état structural est déjà excellent à l'état brut d'hypertrempe, a une résistivité à $4 \mathrm{~K}$ qui passe de 6400 à $10000 \mu \Omega \mathrm{cm}$ par recuit d'une heure à $700^{\circ} \mathrm{C}[23]$.

Les échantillons i-Al-Cu-Fe et i-Al-Cu-Ru bruts d'hypertrempe contiennent, en plus d'une densité de défauts plus forte que dans i-Al-Pd-Mn, une faible proportion d'une seconde phase plus conductrice qui a un effet important sur les phénomènes de transport. Ces phases s'éliminent au recuit, en même temps que les défauts: dans i-Al-Cu-Fe un recuit de 2 heures à $800^{\circ} \mathrm{C}$ multiplie $\rho_{4 \mathrm{~K}}$ par un facteur 3 .

En ce qui concerne i-Al-Cu-Ru, les caractéristiques exceptionnelles obtenues par Biggs et al. $[24]$ ont nécessité des précautions supplémentaires: les auteurs signalent qu'il est essentiel d'effectuer un 
décapage des surfaces par bombardement ionique afin d'éliminer des phases de contamination qui affectent des épaisseurs de l'ordre du micron.

Un autre exemple est fourni par le système Al-Mn-Si. La phase icosaédrique i-Al-Mn-Si, métastable, a une forte densité de défauts qui ne s'éliminent pas par recuit et la valeur maximum de $\rho_{300 \mathrm{~K}}$ atteinte jusqu'à présent est de l'ordre de $800 \mu \Omega \mathrm{cm}$ pour la composition $\mathrm{Al}_{74} \mathrm{Si}_{4} \mathrm{Mn}_{22}$ [9]. Son approximant périodique la phase $\alpha$ - $\mathrm{Al}_{72,5} \mathrm{Mn}_{17,4} \mathrm{Si}_{10,1}$ a une résistivité et un coefficient de température qui varient considérablement avec le traitement thermique parce que les échantillons contiennent des traces d'Al à peine détectables par diffraction des rayons $X(<2 \%)$ qui ne s'éliminent complètement qu'au-dessus de $600^{\circ} \mathrm{C}$. Un traitement thermique adéquat augmente $\rho_{4 \mathrm{~K}}$ jusqu'à $6700 \mu \Omega \mathrm{cm}$ [9], valeur comparable à celles des phases icosaédriques stables de haute perfection structurale.

\subsubsection{Effet de la composition}

Quel que soit l'alliage, le domaine monophasé de la phase icosaédrique stable est toujours très limité en concentration. A l'intérieur de ce domaine la résistivité est très sensible à la composition: Dans i-Al-Cu$\mathrm{Fe}$, un changement de composition de $0,5 \%$ double la conductivité électrique à basse température [27]. Une telle sensibilité rend difficile la comparaison avec les phases périodiques approximantes: la seule comparaison valable doit s'effectuer sur des échantillons strictement monophasés de structure différente mais de même composition. De telles conditions sont réalisables dans le système $\mathrm{Al}-\mathrm{Cu}-\mathrm{Fe}$ où la phase icosaédrique a été comparée à son approximant rhomboédrique ( $\mathrm{a}=32,14 \AA, \alpha=36^{\circ}$, plus de 600 atomes par maille): toutes les mesures effectuées montrent que les propriétés liées aux phénomènes de transport électronique sont identiques pour les deux types d'échantillons [27]. L'ordre à longue distance quasipériodique n'est donc pas nécessaire pour observer une faible conductivité, les propriétés anormales de transport électronique sont imposées par un ordre local qui, si on se réfère aux résultats obtenus sur $\alpha$ $\mathrm{Al}_{72,5} \mathrm{Mn}_{17,4} \mathrm{Si}_{10,1}(\mathrm{a}=12,7 \AA)$, s'étend sur une échelle de longueur de 10 à $20 \AA$.

\subsubsection{Les diagrammes de phases}

On vient de voir la nécessité d'une étude minutieuse du diagramme de phases. Les systèmes les mieux connus à l'heure actuelle sont Al-Pd-Mn [20] et Al-Cu-Fe [28] [29].

Dans le système $\mathrm{Al}-\mathrm{Cu}-\mathrm{Fe}$, plusieurs approximants stables à $700^{\circ} \mathrm{C}$ ont été récemment identifiés et localisés dans un domaine de composition très proche de la phase icosaédrique [30]: ces approximants peuvent s'obtenir monophasés et sont alors clairement identifiés par diffraction des rayons X (figure4). En-dessous de $650^{\circ} \mathrm{C}$ seuls l'approximant thomboédrique et la phase icosaédrique sont encore stables.

Dans Al-Pd-Mn comme dans Al-Cu-Fe, les sections verticales des diagrammes de phases montrent que, en-dessous de $700^{\circ} \mathrm{C}$, le domaine de stabilité de la phase icosaédrique diminue avec la température et il est de plus en plus difficile d'obtenir des échantillons monophasés. A basse température la phase icosaédrique a donc une stoechiométrie déterminée, voisine de $\mathrm{Al}_{62} \mathrm{Cu}_{25,5} \mathrm{Fe}_{12,5}$ et de $\mathrm{Al}_{71} \mathrm{Pd}_{21} \mathrm{Mn}_{8}$.

Cette caractéristique des diagrammes de phases autour de la phase icosaédrique rend délicate l'étude de la stabilité thermique: à basse température la précipitation des phases voisines (approximantes ou non) peut être retardée par une mobilité atomique insuffisante et la phase icosaédrique sursaturée peut évoluer alors vers des états imparfaits ou des structures approximantes métastables. Dans $\mathrm{Al}_{63,5} \mathrm{Cu}_{24} \mathrm{Fe}_{12,5}$, Menguy et al. ont observé en microscopie électronique in situ les différentes étapes d'une transition réversible entre états icosaédrique et microcristallisé rhomboédrique [31].

Dans $\mathrm{Al}-\mathrm{Pd}-\mathrm{Re}$, les propriétés de transport électronique sont très sensibles à la température de traitement thermique, sans qu'une évolution quelconque du spectre de diffraction puisse être détectée: un court recuit à $600^{\circ} \mathrm{C}$ d'un échantillon $\mathrm{Al}_{70} \mathrm{Pd}_{20} \mathrm{Re}_{10}$ préalablement recuit à $800^{\circ} \mathrm{C}$ double la valeur de la résistivité à basse température. Cette observation suggère des effets d'ordre, une transition à haute température vers des états plus désordonnés, liée peut-être au phénomène de percolation des phasons dynamiques prévu théoriquement par Kalugin et Katz [32]. Cette transition s'accompagnerait d'une augmentation brutale du coefficient de diffusion atomique et serait responsable, sur le plan mécanique, de la transition fragile-ductile [33]. 


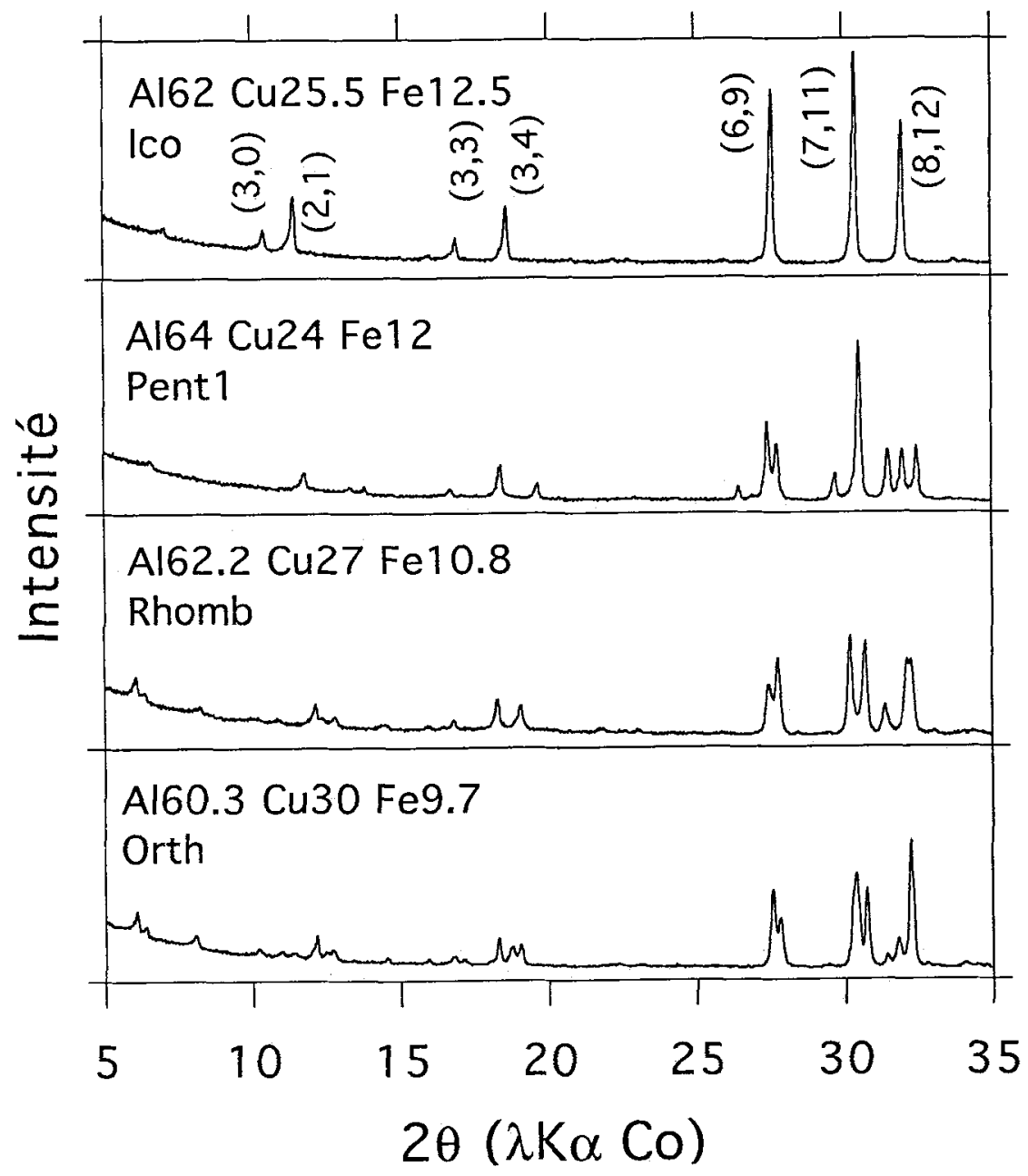

Figure 4: Diagramme de diffraction des rayons $\mathrm{X}$ de i-Al-Cu-Fe et de ses approximants stables a $700{ }^{\circ} \mathrm{C}$ (structures pentagonale, rhomboedrique et orthorhombique). Voir référence [30].

\subsection{Les propriétés mécaniques des phases icosaédriques}

Les quasicristaux se caractérisent par des valeurs de dureté extrêmement élevées pour des alliages d'aluminium: la dureté Vickers est de l'ordre de $1000 \mathrm{~kg} / \mathrm{mm}^{2}$ pour i-Al-Pd-Mn, i-Al-Cu-Fe et i-Al-Cu$\mathrm{Ru}$ et de $500 \mathrm{~kg} / \mathrm{mm}^{2}$ pour i-Al-Li-Cu. Cette dureté s'accompagne d'une très grande fragilité à température ambiante. Par contre, à haute température le comportement devient du type superplastique, avec des taux de déformation qui atteignent $130 \%$ et aucun stade de durcissement structural: Bresson et Gratias ont été les premiers à mettre en évidence un caractère superplastique en observant une diminution de la contrainte d'écoulement quand la déformation augmente [33]. Wollgarten et al. [34] ont montré ensuite que les monograins eux-mêmes avaient ce comportement superplastique.

Le mécanisme de la déformation n'est pas encore compris. Les expériences de Wollgarten et al. ont cependant apporté deux résultats très importants:

- il y a création de dislocations au cours de la déformation plastique [34]

- les dislocations sont mobiles à haute température [35]

deux résultats qui n'étaient pas évidents puisque le mouvement de la dislocation requiert des réarrangements locaux des amas atomiques. Un mécanisme de diffusion facile propre aux quasicristaux intervient donc vraisemblablement quand la ductilité apparait. 


\section{CONCLUSION}

Cette revue rapide de la métallurgie des phases icosaédriques a voulu simplement décrire l'évolution du matériau depuis la découverte et montrer l'importance particulière des conditions de préparation et de caractérisation pour le développement des études fondamentales et pour l'optimisation des propriétés physiques. Ce faisant, elle s'est attachée à souligner les caractères spécifiques qui font des quasicristaux et de leurs approximants - une nouvelle classe de matériaux dont les caractéristiques et les propriétés n'ont pas fini de nous surprendre.

\section{Références}

[1] Quasicrystals: The State of the Art, Directions in Condensed Matter Physics, Vol. 11, D. P. Vincenzo et P. J. Steinhardt Eds. (World Scientific, Singapore, 1991)

[2] Janot C., Quasicrystals: a primer (Oxford Science Publication, 1992)

[3] Lectures on Quasicrystals, F. Hippert et D. Gratias Eds. (Les Editions de Physique, Les Ulis, 1994)

[4] Shechtman S., Blech I., Gratias D. et Cahn J. W., Phys. Rev. Lett. 53 (1984) 1951-1953.

[5] Duneau M. et Katz A., Phys. Rev. Lett. 54 (1985) 2688-2691.

[6] Cahn J. W., Shechtman S. et Gratias D., J. Mater. Res. 1 (1986) 13-26.

[7] Cahn J. W., Gratias D. et Mozer B., J. Phys. France 49 (1988) 1225-1233.

[8] Tsai A.P., Inoue A. et Masumoto T., Jap. J. Appl. Phys. 26 (1987) L1505-L1507.

[9] Biggs B. D., Pierce F. S. et Poon S. J. , Europhys. Lett.. 19 (1992) 415-421.

[10] Guyot P. et Audier M., Phil. Mag. B 52 (1985) L15-L19.

[11] Sainfort P. et Dubost B., J. de Phys. Paris, Col. C3 47 (1986) 321-330.

[12] Audier M., Sainfort P. et Dubost B., Phil. Mag. B 54 (1986) L105-L111.

[13] Donnadieu P., Wang K., Degand C. et Garoche P., J. Non-Cryst. Solids 183 (1995) 100-108.

[14] Stephens P. W. et Goldman A. I., Phys. Rev. Lett. 56 (1986) 1168-1171.

[15] Henley C. L. in ref. [1] pp. 429-524.

[16] Devaud J., Quiquandon M. et Gratias D., "Analysis of dislocations in icosahedral Al-Cu-Fe alloy", Quasicrystals and Incommensurate Structures in Condensed Matter, Mexico 1989, J. Yacaman, D.

Romeu, V. Castano, A. Gomez Eds. (World Scientific, Singapore, 1990) pp.498-515.

[17] Devaud J., Quivy A., Calvayrac Y., Quiquandon M. et Gratias D., Phil. Mag. B 60 (1989) 855-869.

[18] Yokoyama Y., Tsai A. P., Inoue A. et Masumoto T., Mater. Trans. JIM 32 (1991) 1089-1097.

[19] Yokoyama Y., Miura T., Tsai A. P., Inoue A. et Masumoto T., Mater. Trans. JIM 33 (1992) 97-101.

[20] Gödecke T. et Lück R., Z. Metallkd. 86 (1995) 109-121.

[21] Kycia S. W., Goldman A. I., Lograsso T. A., Delaney D. W., Black D., Sutton M., Dufresne E., Brüning R. et Rodricks B., Phys. Rev. B 48 (1993) 3544-3547.

[22] Klein T., Berger C., Mayou D. et Cyrot-Lackmann F., Phys. Rev. Lett. 66 (1991) 2907-2910.

[23] Lanco P., Klein T., Berger C., Cyrot-Lackmann F., Fourcaudot G. et Sulpice A., Europhys. Lett. 18 (1992) 227-232.

[24] Biggs B. D., Poon S. J. et Munirathnam N. R., Phys. Rev. Lett.. 65 (1990) 2700-2703.

[25] Pierce F. S., Guo Q. et Poon S. J., Phys. Rev. Lett.. 73 (1994) 2220-2223.

[26] Honda Y., Edagawa K., Yoshikawa A., Hashimoto T. et Takeuchi S., Jpn. J. App. Phys. 33 (1994) 4929.

[27] Berger C., Gignoux C., Tjernberg O., Lindqvist P., Cyrot-Lackmann F, et Calvayrac Y., Physica B 204 (1995) 44-50.

[28] Gayle F. W., Shapiro A. J., Biancaniello F. S. et Boettinger W. J., Metal. Trans. A 23 (1992) 24092417.

[29] Faudot F., Ann. Chim. Fr. 18 (1993) 445-456.

[30] Quiquandon M., Quivy A., Devaud J., Faudot F., Lefebvre S., Bessière M. et Calvayrac Y., J. Phys. Condens. Matter (soumis).

[31] Menguy N., Audier M., Guyot P. et de Boissieu M., J. Phys. IV France 4 (1994) C3-169-174.

[32] Kalugin P. A. et Katz A., Europhys. Lett. 21 (1993) 921-926.

[33] Bresson L. et Gratias D., J. Non-Cryst. Solids 153 et 154 (1993) 468-472.

[34] Wollgarten M., Beyss M., Urban K., Liebertz H. et Köster U., Phys. Rev. Lett.. 71 (1993) 549-552.

[35] Wollgarten M., Bartsch M., Messerschmidt U., Feuerbacher M., Rosenfeld R., Beyss M. et Urban K., Phil. Mag. Lett. 71 (1995) 99-105. 